\title{
GCU
}

Glasgow Caledonian

University

University for the Common Good

\section{The effect of fake tan on hand hygiene practice}

Melone, Lynn; Price, Lesley; Ness, Valerie; Simpson, Liz; MacDonald, Jennifer; Reilly, Jacqui

Published in:

Journal of Infection Prevention

DOI:

$10.1177 / 1757177418769380$

Publication date:

2018

Document Version

Author accepted manuscript

Link to publication in ResearchOnline

Citation for published version (Harvard):

Melone, L, Price, L, Ness, V, Simpson, L, MacDonald, J \& Reilly, J 2018, 'The effect of fake tan on hand hygiene practice', Journal of Infection Prevention, vol. 19, no. 6, pp. 266-269. https://doi.org/10.1177/1757177418769380

\section{General rights}

Copyright and moral rights for the publications made accessible in the public portal are retained by the authors and/or other copyright owners and it is a condition of accessing publications that users recognise and abide by the legal requirements associated with these rights.

Take down policy

If you believe that this document breaches copyright please view our takedown policy at https://edshare.gcu.ac.uk/id/eprint/5179 for details of how to contact us. 
The Effect of Fake Tan on Hand Hygiene Practice

Lynn Melone ${ }^{1}$, Lesley Price ${ }^{1}$, Valerie Ness ${ }^{1}$, Liz Simpson ${ }^{1}$, Jennifer MacDonald ${ }^{1}$, Jacqueline Reilly ${ }^{1}$

${ }^{1}$ School of Health and Life Sciences, Glasgow Caledonian University, Glasgow, Scotland, UK

\section{Corresponding author}

Lynn Melone, School of Health and Life Sciences, Glasgow Caledonian University, Cowcaddens Road, Glasgow, G4 OBA, UK.

Telephone: +441412731698

Email: Lynn.Melone@gcu.ac.uk 


\begin{abstract}
Hand hygiene is the single most important measure in reducing the spread of infection. The aim of this study was to assess the uptake of fake tan in nursing students and evaluate the impact of wearing fake tan on hand hygiene training lotion removal during handwashing with soap and water. Of the 217 participants recruited, $21 \%$ wore fake tan. Statistical analysis revealed no significant difference between fake tan and non-fake tan wearers. Consequentially, no guidelines regarding the wearing of fake tan in clinical practice are currently required, however the paper identifies what questions remain to be addressed.
\end{abstract}

\title{
Keywords
}

hand hygiene, nursing, infection control, fake tan

\section{Background}

The concept of protecting those in our care is a keystone for patient safety globally and the technique of effective hand hygiene has been shown to be significant in breaking the chain of infection (Jumaa, 2005). A number of factors are recognised for compromising effective hand hygiene; wearing rings, use of nail varnish or false nails or excessively long nails (WHO, 2009). One study has reported that the application of hand lotion significantly increased Staphylococcus aureus carriage immediately after application (Fagernes and Lingass, 2011). However, the impact of the application of another product, fake tan, on hand hygiene remains unknown. This issue was raised by Nursing Lecturers at the authors' University, who observed that during hand hygiene training, nursing students wearing fake tan were unable to remove hand hygiene training lotion from their hands using soap and water. We were therefore interested to conduct a study to explore the impact of wearing fake tan on nursing students' ability to effectively remove hand hygiene training lotion within the simulation learning environment setting. The objectives of this study were to assess the uptake of fake tan in nursing students and to evaluate the impact of wearing fake tan on the removal of hand hygiene training lotion during handwashing with soap and water.

\section{Methods}




\section{Participants and Recruitment}

Ethical approval for the study was obtained from the Research Ethics Committee. Permission was sought and granted from the Head of the Nursing Department to access nursing students on the undergraduate adult field programme, who were then emailed, an invitation to participate in the study, the participant information sheet and consent form. Following the email invitation, lecturing staff asked for volunteer participants and allocated a date and time during timetabled hours for participants to take part. Prior to written consent being obtained, the researchers checked the participants' understanding of the study, the eligibility criteria against the inclusion and exclusion criteria and gave the participants the opportunity to ask any questions. The inclusion criterion was all nursing students willing to take part and the exclusion criterion was those with an active skin condition on their hands. Participants were assigned a study number to anonymise the data.

\section{Data collection}

Participants were asked to remove rings or watches and apply a hand hygiene lotion containing a fluorescent dye to their hands using the World Health Organization's (WHO) six-step technique (WHO 2009 - Figure I). A researcher observed and documented the hand coverage with the training lotion using an ultraviolet (UV) light box. Participants' were then asked to wash their hands with soap and water using the WHO six-step technique and again the researcher observed and recorded how well the training lotion had been removed using the UV light box. To ensure intervention fidelity, a diagram of the WHO six-step technique was displayed to participants and the researcher demonstrated each step of the technique at the same time as the participants applied the training lotion or washed their hands.

\section{Data handling and confidentiality}

The data collected was all fully anonymised and all electronic data was password protected and only available to the researchers. Hard copies of the consent and data collection forms were stored securely but in separate locations and only available for the researchers to access.

Data analysis

Descriptive statistics, including the number and percentage of participants wearing fake tan, the percentage of hand coverage with training lotion before and after hand 
washing and the percentage of training lotion remaining after hand washing were all calculated. The percentage of training lotion removed was calculated by dividing the difference in total surface area covered in training lotion before and after handwashing $\left(\mathrm{cm}^{2}\right)$ by the total surface area covered in training lotion $\left(\mathrm{cm}^{2}\right)$. The percentage of training lotion remaining was then grouped in to the following categories: $0 \%$; <5\%; 5-15\%; >15\%. The association between fake tan use and removal of training lotion was explored using Fisher's exact test in SPSS software (version 22) because assumptions of chi-squared $\left(X^{2}\right)$ analysis were violated due to low frequencies in some cells. Following this analysis, we also dichotomised the estimated percentage of training lotion remaining after handwashing, in to two categories, low $(<5)$ and high $(\geq 5)$ using chi-squared analysis.

\section{Results}

From the 239 participants who agreed to take part in the study, 21 were excluded due to having an active skin condition and one participant was recruited by the researcher but the data collection tool was incomplete, resulting in the 217 included in the analysis. Of the $217,46(21 \%)$ were wearing fake tan.

In both fake tan and non-fake tan wearers, the estimated percentage of training lotion remaining most commonly fell in to the $<5 \%$ grouping. The Fisher's exact test revealed no significant difference between fake tan and non-fake tan wearers in the estimated percentage of training lotion remaining after hand washing in all four categories (Fischer exact test 4.152, $n=217, p=0.243$ ) (Table I).

Moreover, the chi-squared analysis of the two categories, low $(<5)$ and high $(>5)$, also showed no significant difference between fake tan and non-fake tan wearers $\left(X^{2}\right.$ 0.398, $n=217, p=0.563$ ). Post-hoc power calculations in $\mathrm{G}^{*}$ power indicated that for these analyses the study was adequately powered at an alpha of 0.05 (Faul et al, 2007).

\section{Discussion}

This study has shown that wearing fake tan makes no difference to the removal of hand hygiene training lotion from nursing students hands in the simulation learning environment of the clinical skills laboratory, during handwashing with soap and water using the WHO six-step technique (WHO 2009). It therefore does not substantiate the anecdotal reports that fake tan affects the removal of the training lotion. 
Furthermore, this study has also revealed that the uptake of fake tan in this cohort of nursing students was higher than the national average of $15 \%$ in Scotland and $17 \%$ in the UK (Duckett, 2015). However, the difference in the uptake of fake tan might be explained by the age of nursing students, as fake tan has been reported to be more commonly used in 16-34 year olds (Duckett, 2015).

There were several strengths of this study including, it addressing a novel problem identified during clinical simulation practice and it is also an adequately powered study. Furthermore, the data collectors were trained in the measurement of hand coverage which enhanced reliability. It is possible that there could have been differences in the ability of fake tan and non-fake tan wearers to perform the WHO six-step hand hygiene technique. However, this risk of selection bias was minimised by measures to ensure the hand hygiene technique was performed correctly.

This study has shown that currently there is no need for guidelines regarding the wearing of fake tan in clinical practice. Nevertheless, there are still questions to be addressed before we know this with certainty as this study has not investigated whether fake tan affects the bacterial load on the hands of healthcare workers. Further research is required to do this.

\section{Acknowledgements}

The authors would like to thank Dr Kareena McAloney for her assistance with the post-hoc power calculation.

Conflicts of interest: The authors declare that there is no conflict of interest.

\section{References}

Duckett J. (2015) It's a wash-out for fake tan as usage drops to just 13\%. Retrieved from http://www.mintel.com/press-centre/beauty-and-personal-care/its-a-wash-outfor-fake-tan-as-usage-drops-to-just-13 .

Fagernes, M. and Lingass, E. (2011) Factors interfering with the microflora on hands: a regression analysis of samples from 465 healthcare workers. J Adv Nurs, 67, 297-307. doi: 10.1111/j.1365-2648.2010.05462.x 
Faul, F., Erdfelder, E., Lang, A-G. and Buchner, A. (2007) G*Power 3: A flexible statistical power analysis program for the social, behavioural, and biomedical sciences. Behav Res Methods, 39, 175-191. doi:

https://doi.org/10.3758/BF03193146

Jumaa, PA. (2005) Hand hygiene: simple and complex. Int Journal Infect Dis, 9, 314. doi: https://doi.org/10.1016/j.ijid.2004.05.005

World Health Organization (WHO). (2009) WHO Guidelines on Hand Hygiene in Health Care. Retrieved from

http://apps.who.int/iris/bitstream/10665/44102/1/9789241597906 eng.pdf 